\title{
The acute effects of a multi-ingredient pre- workout supplement on resting energy expenditure and exercise performance in recreationally active females
}

\author{
Michael Cameron ${ }^{1}$, Clayton L. Camic ${ }^{2}$, Scott Doberstein ${ }^{1}$, Jacob L. Erickson ${ }^{3}$ and Andrew R. Jagim ${ }^{4^{*}}$ (D)
}

\begin{abstract}
Background: The use of dietary supplements to improve performance is becoming increasingly popular among athletes and fitness enthusiasts. Unfortunately, there is a tremendous lack of research being done regarding female athletes and the use of sport supplements. The purpose of this study was to examine the acute effects of multi-ingredient pre-workout supplement (MIPS) ingestion on resting metabolism and exercise performance in recreationally-active females.

Methods: Fifteen recreationally-active females participated in a randomized, double-blind, placebo controlled study. Subjects completed baseline, and two experimental testing sessions in a cross-over design fashion. Experimental testing included assessment of resting energy expenditure (REE), heart rate, and blood pressure following the ingestion of a MIPS or placebo. Subjects also completed a repetition to failure test for the back squat (BS) and bench press (BP) at $85 \%$ of their 5 -repetition maximum followed by the assessment of anaerobic power using a counter-movement vertical jump test and a sprint test on a force-treadmill. Subjective measurements of energy, focus, and fatigue were also assessed using a 5-point Likert scale. Separate repeated measures analysis of variance (ANOVA) were used to assess differences in REE, cardiovascular responses, and subjective markers between conditions. Performance data were analyzed using paired Student's T-tests.
\end{abstract}

Results: A significant main effect for condition was observed for REE $(p=0.021)$ and diastolic blood pressure $(p=0.011)$ following ingestion of the MIPS. The supplement condition resulted in a greater number of BP repetitions to failure and total work completed during treadmill test $(p=0.039)$ compared to placebo $(p=0.037)$. A significant condition $\mathrm{x}$ time interaction for focus was observed with the supplement treatment exhibiting improved focus at 80-min post ingestion $(p=0.046)$.

Conclusions: Consumption of a MIPS increased resting metabolism following a single dose accompanied by an increase in diastolic blood pressure. Furthermore, acute MIPS ingestion improved upper body muscular endurance and anaerobic capacity while improving feelings of focus following high-intensity exercise in recreationally active females.

Keywords: Thermogenic, Ergogenic aid, Fatigue, Muscular endurance

\footnotetext{
* Correspondence: ajagim@lindenwood.edu

${ }^{4}$ Department of Exercise Science, Lindenwood University, St. Charles, MO

63301, USA

Full list of author information is available at the end of the article
} 


\section{Background}

The use of dietary supplements to enhance exercise performance and improve body composition has long been a popular strategy for active individuals [1]. A newer category of dietary supplements referred to as multi-ingredient pre-workout supplements (MIPS) have gained increased attention among active individuals. These products are a class of supplements that typically include a combination of ingredients such as caffeine, taurine, branch-chain amino acids, creatine, glutamine, and $\beta$-alanine $[2,3]$. MIPS are often manufactured in a proprietary blend and designed to be ingested prior to a workout to enhance exercise performance and potentially lead to enhanced training adaptations over time. Based upon previous findings, the primary active ingredient in most pre-workout supplements appears to be caffeine as it is one of the few ingredients that offers fast-acting performance benefits [4-6], and when ingested alone is still a very popular ergogenic aid for athletes [7]. Caffeine levels peak in the body 30-60 min after ingestion and are widely distributed throughout all tissues [8] exerting its ergogenic effects in a variety of ways. More recent studies have focused on the effects of combining caffeine with a variety of ingredients, including beta-alanine, creatine, and various herbal extracts, for their potential synergistic benefits. Together, these ingredients offer specific physiological advantages, which often include enhanced energy availability, metabolism and improved buffering capacity of skeletal muscle $[4,5,9]$.

Previous studies have reported improvements in acute muscular performance in individuals during upper and lower body exercises following ingestion of MIPS [2, 5, 9, 10]. For example, Beck et al. [9] observed a significant increase in upper body maximal strength in male subjects following acute ingestion of a caffeine-containing supplement. Similarly, Jagim et al. [2] reported a significant improvement in upper body muscular endurance performance following ingestion of a MIPS. In addition to these ergogenic benefits, MIPS, particularly those that contain caffeine, are also marketed as thermogenic agents due to their ability to increase metabolic activity and rates of lipolysis [11-13], potentially resulting in reductions in body weight over time. For example, Acheson et al. [11] observed an increase in metabolic rate after the consumption of caffeine in both individuals who were normal weight and obese. In a more recent study, Campbell et al. [12] reported increases in resting energy expenditure (REE) for up to 3 -h in females who ingested a supplement containing caffeine and green tea extract. Long term, these acute increases in metabolic activity may lead to enhanced fat loss and improvements in body composition as a result of a higher total daily energy expenditure, particularly when combined with specific dietary or activity recommendations.
Manufacturers of MIPS often tend to make claims regarding their product's ability to enhance energy levels and reduce sensations of fatigue during exercise. It is possible that if exercise is more enjoyable and less exhausting, individuals may participate longer, with greater intensity and more frequency; thereby potentially augmenting training adaptations over time. Several reports of benefits ranging from improvements in feelings of focus and energy to reductions in ratings of fatigue following consumptions of a MIPS during bouts of highintensity exercise have been previously reported $[2,5,14]$. Specifically, Walsh et al. [15] reported improved ratings of focus, energy, and decreased feelings of fatigue, which also significantly improved time to exhaustion following consumption of a pre-workout supplement. Similarly, Jagim et al. [2] observed significantly increased subjective feelings of focus with a concomitant reduction in fatigue during a maximal effort sprint test following ingestion of a MIPS.

Although there have been multiple studies supporting the use of MIPS, the majority of the available research to this point has focused primarily on males. There are limited data regarding the effectiveness of pre-workout supplementation in female populations. Recently, a new product that was designed specifically for women has become available, with claims of increasing fat oxidation, increasing energy, and improving performance levels. The product has been shown to be safe for consumption in females [16], however, less is known regarding its influence on metabolism and performance. Therefore, the purpose of the current study was to examine the acute effects of ingesting a MIPS on REE, select clinical health markers, and exercise performance in recreationally active females. A secondary aim was to examine the effects of MIPS ingestion on subjective markers of focus, energy, and fatigue during exercise. It was hypothesized there would be an increase in REE, cardiovascular responses, and exercise performance following ingestion of the MIPS.

\section{Methods \\ Overview}

This study utilized a randomized, double-blind, placebo controlled cross-over design. Subjects first completed a familiarization session to become comfortable with the equipment and testing procedures prior to the experimental sessions. During the familiarization session, subjects completed a demographic form, a Physical Activity Readiness Questionnaire (PAR-Q), and exercise history form. Written consent was also obtained at this time in accordance with the Human Subject Guidelines and approved by the Institutional Review Board at the University of Wisconsin- La Crosse. Subjects reported to the human performance lab within 4-7 days of their 
familiarization session to complete baseline testing which included a body composition assessment, maximal strength testing, and further familiarization trials with the counter movement vertical jump test, and maximal sprint test. The participants were asked to fast for $>2 \mathrm{~h}$ and abstain from exercise $>24 \mathrm{~h}$ prior to baseline testing.

Within 7 days of baseline testing, subjects returned for the first of two experimental testing sessions. Subjects were again asked to fast for $>8 \mathrm{~h}$ and abstain from vigorous exercise $>24 \mathrm{~h}$ prior to experimental testing. Subjects first completed a questionnaire to assess their baseline feelings of focus, energy, and fatigue using a 5-point Likert scale (LS). Subjects then remained seated for a 3-min period followed by the assessment of resting heart rate (HR) and blood pressure (BP). Subjects then ingested either a placebo or the supplement in a randomized and double-blind fashion. Subjects were assessed for changes in REE, HR and BP over a 60 -min period postingestion. At the end of $60 \mathrm{~min}$, a second questionnaire was administered. Following the questionnaire, subjects completed a standardized dynamic warm-up lasting $10 \mathrm{~min}$. Subjects then completed a counter movement vertical jump (CMVJ) test followed by muscular endurance testing using the bench press (BP) and back squat (BS) exercises. Following the muscular endurance testing, a third questionnaire was administered. Subjects rested for a 10-min period and then performed a 25 -s maximal sprint test and completed the questionnaire for a fourth time. Four to seven days following the first testing session, subjects reported to the lab and completed the same protocol, receiving the opposite treatment. Subjects were encouraged to eat similar foods to what they ate prior to each testing session and completed a 2-day dietary history prior to each testing session, which was later assessed for total energy and macronutrient composition.

\section{Subjects}

Fifteen recreationally active college-aged females were recruited to participate in this study (mean $\pm \mathrm{SD}$, age: $21.5 \pm 1.7$ y, height: $165.3 \pm 5.3 \mathrm{~cm}$, weight: $61.6 \pm 5.1 \mathrm{~kg}$, BF\%: $22.9 \pm 4.1 \%$ ). Exclusion criteria included any contradictions to participation which included metabolic disorders, heart disease, arrhythmias, diabetes, thyroid disease, hypertension, hepatorenal, musculoskeletal, autoimmune, or neurological disease. Further exclusion criteria included taking prescription thyroid, cholesterol lowering, diabetic, anti-hypertensive, or anti-inflammatory medications. Inclusion criteria included being recreationally active when regular participation in aerobic and resistance training activities. Recreationally active was defined as participating in at least $150 \mathrm{~min}$ of moderate activity per week for at least six months based on the guidelines by American
College of Sports Medicine [17] (mean \pm SD, hours/week: $5.6 \pm 2.2 \mathrm{~h}$ ). Subjects were instructed to abstain from taking any nutritional supplements and/or ergogenic aids three weeks prior baseline testing, excluding a daily vitamin and/or protein supplementation. The participants were regular caffeine users with a mean caffeine intake value of $175 \pm 26 \mathrm{mg} /$ day. However, caffeine consumption was to be absent within one week of baseline testing until the final testing session.

\section{Testing procedures \\ Familiarization and baseline testing}

During the familiarization session, subjects completed a practice trial for the CMVJ test and a maximal effort sprint test. Subjects then performed 10 repetitions of the $\mathrm{BP}$ and $\mathrm{BS}$ exercises to familiarize themselves with the equipment. Upon arrival to the laboratory for baseline testing, subjects first had their height and body mass determined according to standard procedures using a Healthometer scale (Telstar LLC, Bridgeview, IL). Body composition was then assessed using air displacement plethysmography (BODPOD, Cosmed USA, Inc.). Fat mass percentage values were determined based upon the body densities obtained from the BODPOD. Prior to each testing session, calibration procedures were completed according to the manufacturer guidelines using an empty chamber and a calibrating cylinder of a standard volume $(49.55 \mathrm{~L})$. Subjects were instructed to wear spandex or tight-fitting clothing, remove all jewelry, and wear a swim cap. Following body composition assessment, subjects completed a 10-min dynamic warm-up consisting of $4 \mathrm{~min}$ on a stationary cycle followed by $2 \mathrm{~min}$ of running on a standard treadmill at a speed of $6.0 \mathrm{mph}$ and a set of dynamic stretches including both upper and lower body musculature. Following the warm-up, subjects completed a second CMVJ familiarization trial to get them further accustomed to the movement. Subjects then completed a 5-repetition maximum back squat (5RMBS) and 5-repetition maximum bench press (5RMBP) using an Optima Smith Machine (LifeFitness, Schiller Park, IL). Subjects initially completed a warm-up set of 5 repetitions at approximately $50 \%$ of their estimated 5RM. Next, subjects completed two sets of 5 repetitions at a load corresponding to $60-80 \%$ of their estimated $5 \mathrm{RM}$ with three minutes of rest in between. Subjects then performed subsequent sets of 5 repetitions of increasing weight to determine their 5RM. Three minutes of rest was provided in-between all attempts. All 5RM determinations were made within 1-3 attempts. A successful $5 \mathrm{RMBS}$ was determined by having the participant's thighs parallel with the floor for each repetition. A successful $5 \mathrm{RMBP}$ was determined if the participant lowered the bar to their chest for each repetition. Subjects were encouraged to not pause at the top of each lift for more than a 
second for both 5RMBS and 5RMBP. Five minutes after maximal strength testing, the subjects practiced the maximal sprint test a second time which included two trials (15 s and the second $20 \mathrm{~s}$ ).

\section{Experimental testing Supplementation}

Subjects were instructed to be fasted for $8 \mathrm{~h}$ and restrain from vigorous exercise $>24 \mathrm{~h}$ prior to testing The MIPS tested in this study was a commercially available product supplied by the manufacturer (MusclePharm, Fitmiss ${ }^{\mathrm{mm}}$ Ignite $\left.^{\mathrm{mm}}\right)$. The ingredients are listed in Table 1 . The placebo (PLA) was matched for flavor and color and also provided by the manufacturer. Prior to testing, the assigned condition was prepared by an outside member of the research staff and delivered to the laboratory with the subject's number on a shaker bottle in order to maintain a double blind procedure. Each powder was mixed in a shaker bottle with $16 \mathrm{oz}$. of cold water and a single-serving was ingested within 5 min.

\section{Resting measurements}

Resting energy expenditure and respiratory exchange ratio were assessed using indirect calorimetry and with a TrueOne 2400 metabolic measurement system (ParvoMedics, Sandy, UT). This test is a non-exertional test performed in a fasted state with the subjects lying supine on an exam Table. A clear, hard plastic hood and soft, clear plastic drape was placed over the subjects' head and neck in order to determine resting oxygen uptake and energy expenditure. All subjects laid motionless without falling asleep for the duration of the 60 -min period. The assessment of resting energy expenditure via indirect calorimetry using the TrueOne 2400 metabolic cart system has been shown to be a valid and reliable tool with within subject coefficient of variations ranging from $5.4-10.0 \%$ in male and female populations $[18,19]$. Test to test reliability analysis of this model has yielded a mean intra-class coefficient (ICC) value of $0.942, p<0.001[19]$. Heart rate and blood pressure were

Table 1 Supplement Ingredients

\begin{tabular}{lll}
\hline Serving Size: 1 Scoop (7.2 g) & & \\
\hline Amount Per Serving & Amount & \% Daily Value \\
Calories & 0 & \\
Total Carbohydrates & $1 \mathrm{~g}$ & $<1 \%$ \\
Sugars & $0 \mathrm{~g}$ & $\dagger$ \\
Calcium (as Calcium Silicate) & $34 \mathrm{mg}$ & $3 \%$ \\
Proprietary FitMiss Ignite Blend & $5700 \mathrm{mg}$ & $\dagger$ \\
\hline
\end{tabular}

Other Ingredients:

Carnosyn ${ }^{\oplus}$ Patented Beta Alanine, Choline Bitartrate, L-Tyrosine, L-Glycine, Taurine, L-Carnitine Base, Beet Root Extract (Beta Vulgaris)(High in Nitrates), Hawthorn Berry Powder (Crataegus Pinnatifida)(Fruit), Agmatine Sulfate, Caffeine Anhydrous, Huperzine A 1\% (Huperzia Serrata) also assessed during this time using standard clinical procedures at time points of $15,30,45$, and 60 min post ingestion.

\section{Performance testing}

Following REE assessment, subjects completed the same dynamic warm-up used during baseline testing. Subjects then completed a maximal effort CMVJ. Average power $(\mathrm{W})$ and peak power $(\mathrm{W})$ were later calculated using the participant's body mass $(\mathrm{kg})$, height $(\mathrm{cm})$, and CMVJ height $(\mathrm{cm})$ using previously described procedures [20]. Following the CMVJ test, subjects completed the muscular endurance testing using a resistance set at $85 \%$ of the pre-determined 5RM for the BS and BP respectively. Subjects were instructed to complete as many repetitions as possible during a single set to failure, while completing each repetition as "explosively" as possible without pausing at the top. Subjects were allowed a 5 -min rest period between each exercise. Following a 10-min recovery period, the subjects performed a 25-s maximal sprint test on a non-motorized force treadmill (Woodway, Waukesha, WI, USA) set at a resistance of $12 \%$ of their bodyweight based upon previously used methods [21]. Subjects were given a 3-s count down and were instructed to sprint as fast as possible for the entire 25-s. Sprint tests were analyzed for total work completed, peak and average velocity and power.

\section{Questionnaires}

To assess feelings of focus, energy, and fatigue, a 5-point Likert scale was displayed to them. Specifically, subjects responded verbally to their rating on a scale of $1-5$, which corresponded to: $1=$ low, $2=$ medium-low, $3=$ medium, $4=$ medium-high, $5=$ high as has been previously used [2]. The questionnaire was administered at baseline, 60min post-ingestion, post-bench press and immediately following the sprint test.

\section{Dietary analysis}

Each subject's 2-day diet history was assessed using a commercially available nutrition analysis program (MyFitnessPal, Inc.) to assess differences in total energy and macronutrient intake during a 2-day period prior to each testing session to ensure the outcomes of the study were not influenced by dietary intake.

\section{Statistical analysis}

All data were analyzed using the Statistical Package for the Social Sciences (SPSS Inc., Chicago, IL). Descriptive statistics were used to quantify baseline physical characteristics. Treatment (supplement or placebo) $\mathrm{x}$ time repeated measures analyses of variance (ANOVAs) were used to assess differences in $\operatorname{REE}$ (35, $60 \mathrm{~min}$ ); Likert scale scores $(0,60,80,90 \mathrm{~min})$ and $\mathrm{HR}$ and blood pressure 
(0, 15, 30, 45, 60, minutes). Paired student's T-tests were used to assess differences in muscular endurance and anaerobic performance between each condition. Data were considered statistically significant when the probability of type I error was $p<0.05$. If a significant interaction was observed for the ANOVA, a Tukey's honest significant differences (HSD) post-hoc analysis was performed in order to determine where significance occurred between conditions.

\section{Results}

\section{Resting energy expenditure}

A significant main effect for condition was observed for REE (MIPS: $1497 \pm 55.7$; PLA: $1416 \pm 42 \mathrm{kcal} /$ day $p=0.043)$. Post-hoc analysis revealed the MIPS condition exhibited a higher REE at $35 \mathrm{~min}$ and $60 \mathrm{~min}$ postingestion as seen in Fig. 1. Due to machine complications, one participant's data was discarded from the 35-min data time point.

\section{Hemodynamic variables}

There were no significant differences observed for systolic blood pressure overall or between conditions following ingestion of the MIPS or PLA. There was a significant main effect for time $(p=0.002)$ observed regarding HR responses. Heart rate was significantly higher at baseline compared to each time point post-ingestion in both conditions. There was an overall main effect for condition regarding mean diastolic blood pressure following ingestion of the MIPS compared to the PLA (MIPS: 84 \pm 1.1 ; PLA: $64.8 \pm 1.3 \mathrm{mmHg}, p=0.011)$; however, no significant condition $\mathrm{x}$ time interaction $(p=0.44)$ was observed.

\section{Performance measures}

Subjects completed a significantly higher number of BP repetitions to failure following ingestion of the MIPS condition compared to PLA $(p=0.037)$ as seen in Fig. 2.

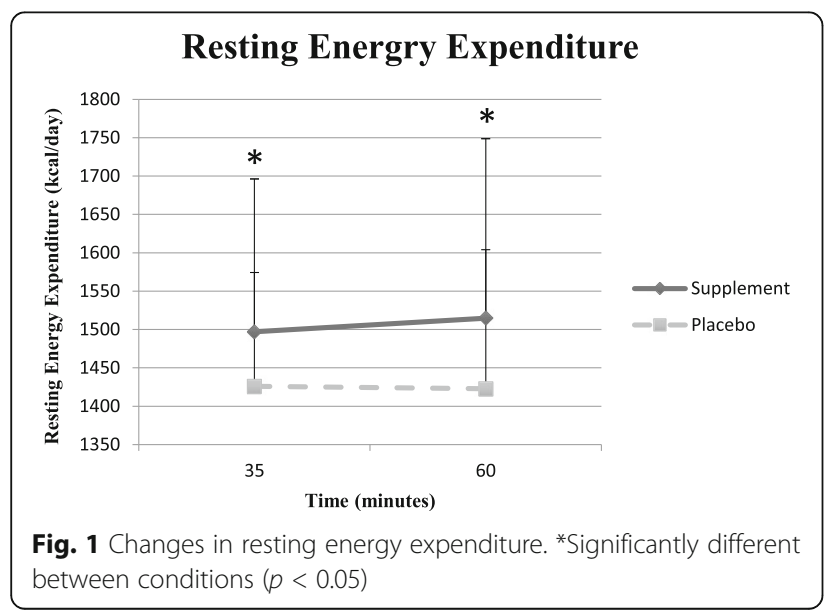

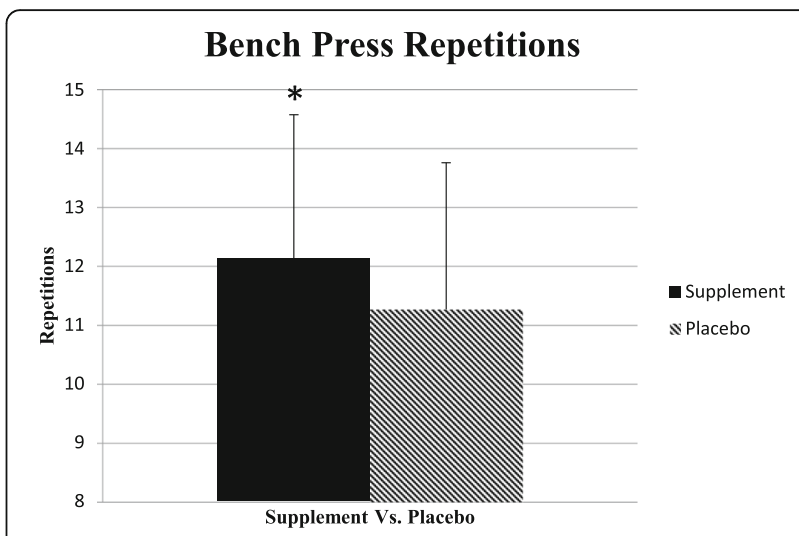

Fig. 2 Mean bench press repetitions to failure. *Significantly different between conditions $(p<0.05)$

There was no significant difference in BS performance between the MIPS and PLA (MIPS: $13.5 \pm 3.7$; PLA: $12.8 \pm 3.1$ reps, $p=0.28$ ). Subjects completed a significantly greater amount of total work $(\mathrm{m})$ during the 25-s treadmill sprint following ingestion of the MIPS compared to the PLA (MIPS: $93.97 \pm 6.63$; PLA: $93.01 \pm 7.49 \mathrm{~m}, p=0.039$ ).

Table 2 provides a summary of CMVJ and treadmill performance variables following ingestion of the drinks.

\section{Dietary analysis}

There were no significant differences between the subject's mean energy and macronutrient intakes prior to each testing session as presented in Table 3.

\section{Side effects and subjective measures}

The side effects reported by the subjects included symptoms of having a "flush face", "tingly hands", and "jitteriness" $(n=4)$ approximately $30 \mathrm{~min}$ post-ingestion of the MIPS but not the PLA. A significant condition $\mathrm{x}$ time interaction was observed regarding feelings of focus. Posthoc analysis revealed MIPS exhibited greater feelings of focus at $80 \mathrm{~min}$ post ingestion as seen in Fig. $3(p=0.046)$.

Table 2 CMVJ and treadmill performance data (mean \pm SD)

\begin{tabular}{lll}
\hline & MIPS $(n=15)$ & Placebo $(n=15)$ \\
\hline Countermovement Vertical Jump & & \\
Height $(\mathrm{cm})$ & $47.3 \pm 3.9$ & $47.3 \pm 3.4$ \\
Average Power $(\mathrm{W})$ & $2012.8 \pm 222.3$ & $2016.3 \pm 208.9$ \\
Peak Power $(\mathrm{W})$ & $3607.8 \pm 451.9$ & $3614.5 \pm 409.7$ \\
Treadmill Performance & & \\
Average Velocity $(\mathrm{m} / \mathrm{s})$ & $3.7 \pm 0.4$ & $3.6 \pm 0.3$ \\
Peak Velocity $(\mathrm{m} / \mathrm{s})$ & $4.3 \pm 0.5$ & $4.2 \pm 0.4$ \\
Average Power $(\mathrm{W})$ & $421.6 \pm 61.1$ & $419.8 \pm 63.5$ \\
Peak Power $(\mathrm{W})$ & $1481.5 \pm 235.7$ & $1454.9 \pm 331.2$ \\
Total Work $(\mathrm{m})(n=14)$ & $94.0 \pm 6.7^{*}$ & $93.01 \pm 7.5$ \\
\hline
\end{tabular}

*Significantly different between conditions $(p<0.05)$ 
Table 3 Summary of the 2-day dietary history for each condition

\begin{tabular}{lll}
\hline Measurement & MIPS $(\mathrm{N}=15)$ & Placebo $(\mathrm{N}=15)$ \\
\hline Calories (kcals/d) & $1597.7 \pm 247.8$ & $1638.1 \pm 243.9$ \\
Carbohydrates $(\mathrm{g})$ & $219.2 \pm 49.26$ & $215.9 \pm 208.9$ \\
Fat $(\mathrm{g})$ & $53.6 \pm 20.9$ & $55.8 \pm 21.6$ \\
Protein $(\mathrm{g})$ & $69.7 \pm 24.0$ & $71.5 \pm 22.9$ \\
\hline
\end{tabular}

Data are presented as Mean \pm SD

There was a main effect for time regarding feelings of energy throughout the workout for both conditions as subjects reported greater energy at 80 min post ingestion compared to energy levels at $90 \mathrm{~min}$ post ingestion $(p<$ $0.01)$. A significant main effect for time regarding feelings of fatigue was observed for both conditions beginning 90 min post-ingestion $(p<0.001)$ however no significant condition $\mathrm{x}$ time interaction was observed $(p>0.05)$.

\section{Discussion}

The purpose of the current investigation was to examine the acute effects of ingesting a commercially available multi-ingredient pre-workout supplement on REE, exercise performance and select markers of clinical health in recreationally active females. The primary findings from the current study indicated that ingestion of a MIPS resulted in an acute increase in REE. These results are in accordance with previous studies that have also observed significant increases in REE following ingestion of a MIPS or caffeine containing supplement [11, 12, 22]. For example, Outlaw et al. [22] reported significant increases in REE following ingestion of a commercially available caffeine-containing supplement (340 $\mathrm{mg}$ of caffeine) for $3 \mathrm{~h}$ post ingestion. Similarly, Campbell et al. [12] reported a significant increase in REE up to three hours post ingestion of a MIPS thermogenic supplement that contained $150 \mathrm{mg}$ of caffeine plus green tea extract in healthy females. However, others have reported no significant changes in metabolic activity following ingestion

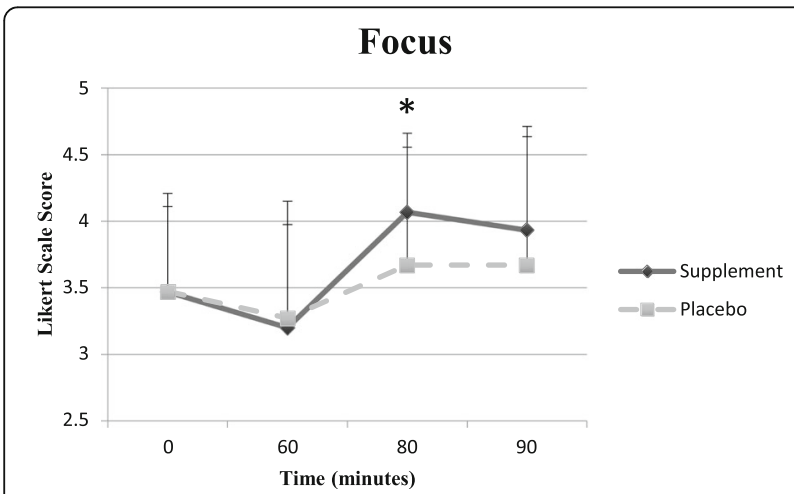

Fig. 3 Changes in ratings of focus. *Significantly different between conditions $(p<0.05)$ of thermogenic agents. As evidenced, Rashti et al. [23] did not observe a significant increase in REE following ingestion of a caffeine-containing supplement. Although, it should be noted that the subjects from the Rashti et al. study [23] were only in a 3-h post absorption state suggesting that increases in REE may only occur during a period of an extended fast $(>3 \mathrm{~h}$ ). It is likely that the increases in REE observed in the current study is a result of the caffeine contained within the product as similar increases in REE have been shown to occur following consumption of caffeine alone [11]. Caffeine acts as an adenosine antagonist, which has a regulatory influence on metabolic activity. Therefore, when caffeine binds to adenosine receptors, it likely increase acute metabolic activity such as the case in the current study. This increase in REE is likely to persist even in those who are regular caffeine users as was also seen in the current study. This is further supported by the aforementioned study done by Outlaw et al. [22] who observed the significant increase in $\mathrm{REE}$ at $60 \mathrm{~min}$ post-ingestion of a caffeine-containing supplement, even though the subjects were regular caffeine consumers and reported average caffeine intakes of approximately $200 \mathrm{mg}$ per day.

Furthermore, results from the current study also indicate that MIPS ingestion does not appear to negatively influence $\mathrm{HR}$ in recreationally active females as HR remained stable and did not significantly increase following consumption of a MIPS compared to a PLA. This finding is consistent with other studies $[3,12]$ which also observed minimal changes in HR following ingestion of a MIPS. Conversely, some studies [24, 25] have reported increases in HR following consumption of a MIPS, which may be attributable to differences in the amount of caffeine consumed or the caffeine habits of the subjects.

Based upon the results of the current study, it also does not appear as though systolic blood pressure is significantly influenced by MIPS ingestion. These findings are in contrast to previous studies, which have observed significant increases in systolic blood pressure following consumption of MIPS or caffeine-containing beverages $[3,12,25]$. For example, Campbell et al. [12] reported a significant increase in both systolic and diastolic blood pressure following ingestion of a caffeine-containing thermogenic supplement in female subjects. Interestingly, a significant increase in only diastolic BP was observed in the current study. These contradictory findings may again be attributable to the differences in caffeine dosage. As stated previously, caffeine is an adenosine antagonist and therefore acts as a vasoconstrictor. This vasoconstriction can lead to an increase in systemic vascular resistance and the isolated rise seen in diastolic pressure in this study may be explained by this as vascular resistance impacts diastolic blood pressure to a greater degree than systolic pressure. A change in diastolic blood pressure of this 
magnitude however is rather abnormal, particularly under resting conditions and warrants further investigation. For the current investigation, the MIPS contained a blend of caffeine and various other ingredients, however, the exact amounts are unknown. Previous studies have observed changes in $\mathrm{HR}$ and systolic BP following ingestion of caffeine-containing beverages with dosages ranging from 230 to $495 \mathrm{mg}$, which may explain some of the inconsistencies regarding hemodynamic responses [3, 12, 24, 25]. Further, acute increases in diastolic blood pressure may be a concern for those with cardiovascular disorders. However, a recent study by Vogel et al. [5] investigated the same MIPS used in the current study and determined it to be safe for females to consume 1 or 2 servings of a MIPS daily for 28 days as evidenced by a lack of change in hematological markers or resting vitals in recreationally active females. Based on the results of the current study and those from Vogel et al. [5], it appears as though the supplement does not result in any serious adverse effects with the exception for the potential rise in diastolic blood pressure.

The current investigation observed a significant increase in upper body muscular endurance, which is in agreement with the previous findings that have investigated the acute effects of MIPS on various aspects of performance $[2,26,27]$. It can be assumed that the caffeine contained within the current MIPS likely played a significant role in the observed performance benefits, as it is one of the few ingredients with an immediate mechanism of action as described earlier and has been previously shown to improve performance [6]. It has been proposed that caffeine may positively influence muscular endurance by its direct effect on muscle anaerobic energy provision and its ability to increase muscle contractility [26]. Further, caffeine also acts as a central nervous stimulant and therefore may delay the onset of fatigue or allow individuals to better tolerate a higher training intensity ultimately allowing for a greater work capacity $[24,26]$. A recent meta-analysis [6] found that caffeine ingestion appears to improve muscular endurance (overall $E S=0.28$, $p<0.01)$ and increase maximum voluntary contraction, particularly during lower body exercises (overall $\mathrm{ES}=0.67$, $p<0.01)$. However, the current investigation did not observe a significant increase in lower body muscular endurance. These findings are in opposition to a prior investigation, which observed a significant increase in lower body endurance, but not upper body [24] following ingestion of a MIPS in males. These differences in performance outcomes may be explained by differences in instrumentation utilized (i.e., leg press versus the back squat) or too low of a caffeine amount to elicit a positive improvement in lower body performance. It is worth noting that other ingredients in the MIPS may also have contributed to the improvement in performance as some of them are designed to also enhance exercise performance. For example, tyrosine supplementation is purported to reduce sensations of central fatigue during strenuous exercise however the supporting evidence is not clear as previous studies have failed to detect any substantial improvements in strength, exercise capacity or anaerobic power $[27,28]$. Beet-root extract is also purported to enhance exercise performance by augmenting the vasodialatory response to exercise thereby improving blood flow. There is some evidence to support its ability to improve exercise performance however, as a result of the proposed mechanism of action, it is not likely to influence the exercise modalities used in the current study in addition to the fact that prior research also suggests that multiple doses overall several days may be required in order to elicit an ergogenic benefit [29-31]. Additionally, it would be expected that if adequate amounts of boot root extract were present, reductions in blood pressure would have occurred as a result of increased vasodilation and antihypertensive responses which is contradictory to the present findings. Similarly, beta-alanine supplementation has also been shown to enhance exercise performance, particularly during bouts of high-intensity exercise lasting $30-180 \mathrm{~s}$. as a result of an improvement in buffering capacity. However, it is again believed that several dosages over the course of multiple weeks ( $\sim$ weeks) is required to substantiate any performance benefits [32]. Additionally, the dosage of beta-alanine required for an ergogenic benefit appears to be $4-6 \mathrm{~g} / \mathrm{d}$ [32] and the MIPS used in the current study only contains $5.6 \mathrm{~g}$ of a proprietary blend which also contains several other ingredients. Therefore, the likelihood of there being enough beta-alanine present to elicit any acute benefit is low. Taurine is another ingredient that has been proposed to enhance anaerobic performance [33]; however, once again, a larger dose $(\sim 5 \mathrm{~g})$ is likely required to substantiate any ergogenic benefit.

In the current investigation ingestion of the MIPS did not appear to influence lower body power, which is in accordance with previous findings. For example, Jagim et al. [2], also did not observe a significant improvement in lower body power following ingestion of a MIPS. However, Jagim et al. [2] did observe a significant increase in mean power during a maximal effort sprint test. Adversely, the current investigation observed a significant increase in total work during the maximal sprint following ingestion of the MIPS but not power. All other anaerobic power measurements during the maximal sprint test were not significantly different following ingestion of a MIPS compared to the PLA, which is similar to results seen in previous studies $[2,8]$.

A secondary aim of the current study was to examine how MIPS ingestion influences subjective markers of focus, energy, and fatigue during exercise. Based upon 
the results of the current investigation, it appears as though acute MIPS ingestion may positively influence feelings of focus following a bout of high-intensity exercise. The findings of the current investigation are in agreement with the observations from a previous study [15] during which subjects reported statistically greater feelings of focus and energy 10-min into running on a treadmill at $70 \%$ of $\dot{\mathrm{V}}_{2}$ max following ingestion of a MIPS. However, the current study did not observe a statistical difference in subjective feelings of energy or fatigue levels post exercise following ingestion of a MIPS compared to PLA. These findings are in opposition of Jagim et al. [2] who reported reductions of fatigue levels throughout testing following ingestion of a MIPS during a strength training protocol. This disparity between the two conditions may be more drastic due to the greater workload of a strength training protocol consisting of 5 sets of 5 repetitions with a 6th set to failure [2]. Additionally, differences in ingredient amounts, particularly caffeine, across different MIPS's may also yield contradictory findings relating to subjective measures of fatigue and energy levels. It is likely that caffeine contained within the MIPS was primarily responsible for the observed increase in focus as previous research has observed similar findings during high-intensity exercise following consumption of caffeine-containing beverages $[34,35]$. Tyrosine, which is also one of the included ingredients, has also been proposed to improve cognitive performance during exercise [36]. For example, Deijen et al. [36] observed improvements in cognitive performance during periods of physical stress in military cadets however a relatively large dose was used in the study and the supplement was ingested for a 6-day period.

\section{Conclusions}

Ingestion of a MIPS appears to result in acute elevations in REE up to 60 min post ingestion in recreationally active females. These elevations appear to occur with minimal changes in heart rate and systolic blood pressure however an increase in diastolic blood pressure was observed and individuals with risk factors for cardiovascular disease may need to exercise caution prior to consuming such a product. Further, the current MIPS appears to positively influence upper body muscular endurance, which could enhance training adaptations over time by allowing for a greater training volume. The added improvement of the subjective measure of focus could further improve the quality of a training session. Additional research is needed to examine the long-term effects of MIPS ingestion on measures of clinical health and training adaptations in female populations.

\section{Acknowledgments}

We would like to thank all of the subjects who participated in the study as well as the research assistants in the Human Performance Laboratory at the University of Wisconsin-La Crosse who helped with data collection.

\section{Funding}

This study was co-funded through an unrestricted education grant from the International Society of Sports Nutrition and MusclePharm Corporation (Denver, CO). We would like to thank MusclePharm for providing the supplement and placebo products that allowed us to conduct the study. MusclePharm Corporation is the manufacturer of Fitmiss ${ }^{T M} \operatorname{lgnite}^{T M}$. The funding agencies had no role in the experimental design used, collection of data, interpretation of results or concluding statements.

\section{Availability of data and materials}

Data and publication materials can be provided upon request. Please contact corresponding author for this information.

\section{Authors' contributions}

MC coordinated data collection, assisted with data analysis and manuscript preparation. CLC assisted with data analysis, interpretation of results and manuscript preparation. SD assisted with interpretation of results. JE assisted with interpretation of results and manuscript preparation. ARJ designed the study, oversaw data collection, analysis and manuscript preparation. All authors approved the final version of this manuscript.

\section{Ethics approval and consent to participate}

Ethics approval for the study was approved by the Institutional Review Board at the University of Wisconsin - La Crosse. All subjects provided written consent before participating in the study.

\section{Consent for publication}

Not applicable.

\section{Competing interests}

The study was funded from the International Society of Sports Nutrition and MusclePharm@. The authors declare they have no competing interests. All researchers involved independently collected, analysed, and interpreted the results from this study and have no financial interests concerning the outcome of the current investigation. The results of this study do not constitute endorsement by the authors and/or the institution concerning the nutritional product of interest.

\section{Publisher's Note}

Springer Nature remains neutral with regard to jurisdictional claims in published maps and institutional affiliations.

\section{Author details}

${ }^{1}$ Exercise \& Sport Science Department, University of Wisconsin - La Crosse, La Crosse, WI, USA. '2Department of Kinesiology and Physical Education, Northern Illinois University, DeKalb, IL 60115, USA. ${ }^{3}$ Mayo Clinic Health Systems, Sports Medicine, Onalaska, WI 54650, USA. ${ }^{4}$ Department of Exercise Science, Lindenwood University, St. Charles, MO 63301, USA.

Received: 1 September 2017 Accepted: 26 December 2017

Published online: 05 January 2018

\section{References}

1. Baron M. Fighting obesity: part 3: over-the-counter weight-loss supplements. Health Care Food and Nutrition. Focus. 2004:21(12):5-7.

2. Jagim $A$, Jones $M$, Wright $G$, Antoine $C$, Kovacs $A$, Oliver $M$. The acute effects of a multi-ingredient pre-workout ingestion on strength performance, lower body power, and anaerobic capacity. Journal of International Society of Sports Nutrition. 2016;13:11.

3. Kedia W, Hofhein J, Habowski S, Ferrando A, Gothard M, Lopex H. Effects of a pre-workout supplement on lean mass, muscular performance, subjective workout experience, and biomarkers of safety. International Journal of Medical Studies. 2014;11(2):116-26.

4. Spriet LL. Caffeine and performance. International journal of sport nutrition. 1995;5(s1):S84-S99.

5. Vogel R, Joy J, Falcone P, Mosman M, Kim M, Moon J. Safety of a doseescualted pre-workout supplement in recreationally active females. Journal of the international Society of Sports Nutrition. 2015;12:12.

6. Warren G, Park N, Maresca R, Mckibans K, Millard-Stafford M. Effect of caffeine ingestion on muscular strength and endurance: a meta-analysis. Med Sci Sports Exerc. 2010;42(7):1375-87. 
7. Antonio J. Caffeine: the forgotten ergogenic aid. Strength \& Conditioning Journal. 2004;26:50-1.

8. Goldstein E, Ziegenfuss T, Kalman D, Kreider R, Campbell B, Wilborn C, Taylor L, Wiloughby D, Stout J, Graves B, Wildman R, Ivy J, Spano M, Smith A, Antonio J. International society of sports nutrition position stand: caffeine and performance. Journal of the International Society of Sports Nutrition. 2010;7:5.

9. Beck TW, Housh TJ, Schmidt RJ, Johnson GO, Housh DJ, Coburn JW, Malek $\mathrm{MH}$. The acute effects of a caffeine-containing supplement on strength, muscular endurance, and anaerobic capabilities. The Journal of Strength \& Conditioning Research. 2006;20(3):506-10.

10. Hoffman JR, Kang J, Ratamess NA, Rashti SL, Tranchina CP, Faigenbaum AD. Thermogenic effect of an acute ingestion of a weight loss supplement. J Int Soc Sports Nutr. 2009;6:1.

11. Acheson K, Zahorska-Markiewicz B, Pittet PH, Anantharaman K, Jequier E. Caffeine and coffee: their influence on metabolic rate and substrate utilization in normal weight and obese individuals. Am J Clin Nutr. 1980;33(5):989-97.

12. Campbell BI, Zito G, Colquhoun R, Martinez N, Kendall K, Buchanan L, Lehn M, Johnson M, St. Louis C, Smith Y, Cloer B, Pingel A. The effects of a singledose thermogenic supplement on resting metabolic rate and hemodynamic variables in healthy females - a randomized, double-blind, placebo-controlled, cross-over trial. Journal of the International Society of Sports Nutrition. 2016;13(1):13.

13. Dalbo VJ, Roberts MD, Stout JR, Kerksick CM. Acute effects of ingesting a commercial thermogenic drink on changes in energy expenditure and markers of lipolysis. Journal of the International Society of Sports Nutrition. 2008;5(1):6.

14. Duncan M, Smith M, Cook K, James R. The acute effect of a caffeinecontaining energy drink on mood state, readiness to invest effort, and resistance exercise to failure. Journal of Strength and Conditioning Research. 2012;26(10):2858-65.

15. Walsh A, Gonzalez A, Ratamess JK, Hoffman JR. Improved time to exhaustion following ingestion of the energy drink amino impact. Journal of the International Society of Sports Nutrition. 2010;7:14.

16. Vogel RM, Joy JM, Falcone P, Mosman M, Kim M, Moon JR. Safety of a doseescalated pre-workout supplement in recreationally active females. J Int Soc Sports Nutr. 2015;12:12.

17. Pescatello L, Arena R, Riebe D, Thompson P. ACSM's quidelines for exercise testing and prescription: American college of sports medicine. Philadelphia: Lippincott, Williams \& Wilkins; 2014.

18. Cooper JA, et al. Assessing validity and reliability of resting metabolic rate in six gas analysis systems. J Am Diet Assoc. 2009;109(1):128-32.

19. Kerksick C, et al. Effects of a popular exercise and weight loss program on weight loss, body composition, energy expenditure and health in obese women. Nutr Metab (Lond). 2009:6:23.

20. Johnson DL, Bahamonde R. Power output estimate in university athletes. Journal of Strength and Conditioning Research. 1996;10(3):161-6.

21. McClain T, Wright G, Camic C, Kovacs A, Hegge J, Brice G. Development of an anaerobic sprint running test using a nonmotorized treadmill. Journal of Strength and Conditioning Research. 2015;29(8):2197-204.

22. Outlaw J, Wilborn C, Smith A, Urbina S, Hayward S, Foster C, Taylor L. Effects of ingestion of a commercially available thermogenic dietary supplement on resting energy expenditure, mood state and cardiovascular measures. Journal of the International Society of Sports Nutrition. 2013;10(1):25.

23. Rashti S, Ratamess N, Kang J, Faigenbaum A, Chilakos A, Hoffman J. Thermogenic effect of meltdown RTD ${ }^{\mathrm{TM}}$ energy drink in young health women: a double blind, cross-over design study. Lipids Health Dis. 2009;8:57.

24. Spradley BD, Crowley KR, Tai CY, Kendall KL, Fukuda DH, Esposito EN, Moon JR. Ingesting a pre-workout supplement containing caffeine, B-vitamins, amino acids, creatine, and beta-alanine before exercise delays fatigue while improving reaction time and muscular endurance. Nutr Metab (Lond). 2012;9:28.

25. Hoffman JR, Kang J, Ratamess A, Hoffman M, Tranchina C, Faigenbaum A. Examination of a pre-exercise, high energy supplement on exercise performance. Journal of the International Society of Sports Nutrition. 2009;6:2.

26. Gonzalez AM, Walsh AL, Ratamess NA, Kang J, Hoffman JR. Effect of a preworkout energy supplement on acute multi-joint resistance exercise. Journal of sports science \& medicine. 2011;10(2):261.

27. Chinevere TD, Sawyer RD, Creer AR, Conlee RK, Parcell AC. Effects of Ltyrosine and carbohydrate ingestion on endurance exercise performance. J Appl Physiol. 2002;93(5):1590-7.
28. Sutton EE, Coll MR, Deuster PA. Ingestion of tyrosine: effects on endurance, muscle strength, and anaerobic performance. Int I Sport Nutr Exerc Metab. 2005;15(2):173-85

29. Muggeridge DJ, Howe CC, Spendiff O, Pedlar C, James PE, Easton C. A single dose of beetroot juice enhances cycling performance in simulated altitude. Med Sci Sports Exerc. 2014;46(1):143-50.

30. Cermak NM, Gibala MJ, Van Loon L. Nitrate supplementation's improvement of 10-km time-trial performance in trained cyclists. International journal of sport nutrition and exercise metabolism. 2012;22(1):64-71.

31. Cermak NM, Res P, Stinkens R, Lundberg JO, Gibala MJ, van Loon L. No improvement in endurance performance after a single dose of beetroot juice. International journal of sport nutrition and exercise metabolism. 2012;22(6):470-8.

32. Trexler ET, Smith-Ryan AE, Stout JR, Hoffman JR, Wilborn CD, Sale C, et al. International society of sports nutrition position stand: Beta-alanine. Journal of the International Society of Sports Nutrition. 2015;12(1):30.

33. Warnock $R$, Jeffries $\mathrm{O}$, Patterson $\mathrm{S}$, Waldron $\mathrm{M}$. The effects of caffeine, taurine or caffeine-taurine co-ingestion on repeat-Sprint cycling performance and physiological responses. Int J Sports Physiol Perform. 2017;24:1-20.

34. Hoffman JR, Kang J, Ratamess NA, Hoffman MW, Tranchina CP, Faigenbaum AD. Examination of a pre-exercise, high energy supplement on exercise performance. Journal of the International Society of Sports Nutrition. 2009:6(1):2.

35. Hogervorst E, Riedel WJ, Kovacs E, Brouns FJPH, Jolles J. Caffeine improves cognitive performance after strenuous physical exercise. Int J Sports Med. 1999;20(06):354-61.

36. Deijen JB, Wientjes CJE, Vullinghs HFM, Cloin PA, Langefeld JJ. Tyrosine improves cognitive performance and reduces blood pressure in cadets after one week of a combat training course. Brain Res Bull. 1999;48(2):203-9.

\section{Submit your next manuscript to BioMed Central and we will help you at every step:}

- We accept pre-submission inquiries

- Our selector tool helps you to find the most relevant journal

- We provide round the clock customer support

- Convenient online submission

- Thorough peer review

- Inclusion in PubMed and all major indexing services

- Maximum visibility for your research

Submit your manuscript at www.biomedcentral.com/submit
( Biomed Central 